\title{
Unique synthesis of graphene-based materials for clean energy and biological sensing applications
}

\author{
XU MingSheng, GAO Yan, YANG Xi \& CHEN HongZheng* \\ State Key Laboratory of Silicon Materials, Key Laboratory of Macromolecule Synthesis and Functionalization, Ministrg of Education, \\ Department of Polymer Science and Engineering, Zhejiang University, Hangzhou 310027, China
}

Received December 27, 2011; accepted February 2, 2012; published online April 28, 2012

\begin{abstract}
Graphene has unique physical properties, and a variety of proof-of-concept devices based on graphene have been demonstated. A prerequisite for the application of graphene is its production in a controlled manner because the number of graphene layers and the defects in these layers significantly influence transport properties. In this paper, we briefly review our recent work on the controlled synthesis of graphene and graphene-based composites, the development of methods to characterize graphene layers, and the use of graphene in clean energy applications and for rapid DNA sequencing. For example, we have used Auger electron spectroscopy to characterize the number and structure of graphene layers, produced single-layer graphene over a whole Ni film substrate, synthesized well-dispersed reduced graphene oxide that was uniformly grafted with unique gold nanodots, and fabricated graphene nanoscrolls. We have also explored applications of graphene in organic solar cells and direct, ultrafast DNA sequencing. Finally, we address the challenges that graphene still face in its synthesis and clean energy and biological sensing applications.
\end{abstract}

graphene, clean energy, chemical and biological sensors, synthesis, chemical vapor deposition, surface segregation, organic solar cells, DNA sequencing

Citation: $\quad$ Xu M S, Gao Y, Yang X, et al. Unique synthesis of graphene-based materials for clean energy and biological sensing applications. Chin Sci Bull, 2012, 57: 3000-3009, doi: 10.1007/s11434-012-5128-9

Graphene is an atomically thin layer of $\mathrm{sp}^{2}$ hybridized carbon atoms arranged in a honeycomb lattice [1]. It is the fundamental building block for all $\mathrm{sp}^{2}$-hybridized carbon allotropes (e.g., graphite, nanotubes and fullerenes). Observation of graphitic monolayers by chemists and surface scientists dates back to the 1960s [2,3]. In 1986, Boehm et al. [4] referred to such a single layer of graphite as graphene. However, exfoliation of graphite into individual graphene sheets remained a curiosity until 2004, when graphene sheets isolated using the simple Scotch tape method were first reported [5,6]. The subsequent discovery of its unusual electronic properties has led to an extraordinary amount of interest amongst researchers across virtually all scientific disciplines [7-10]. Graphene has a large theoretical specific surface area $\left(2630 \mathrm{~m}^{2} \mathrm{~g}^{-1}\right)$ [11], high intrinsic mobility (200000 $\mathrm{cm}^{2} \mathrm{~V}^{-1} \mathrm{~s}^{-1}$ ) [12], high Young's modulus ( 1.0 TPa) [13],

*Corresponding author (email: hzchen@zju.edu.cn) good thermal conductivity $\left(\sim 5000 \mathrm{~W} \mathrm{~m}^{-1} \mathrm{~K}^{-1}\right)$ [14] and optical transmittance ( 97.7\%) [15]. Graphene is thus considered as an attractive candidate for next-generation electronic materials and holds great promise for widespread applications [16-24] including ultrafast optoelectronic devices, chemical and biological sensors, energy generation and storage, and novel composites.

As the first step to investigate its properties and further to realize these practical applications, graphene must be synthesized in a controlled manner, so the controlled synthesis of graphene is of great importance for both fundamental research and practical applications [25]. Recent synthetic efforts have diverged in a number of different directions. For instance, micromechanical cleavage [6], liquid-phase exfoliation [26,27], reduction of graphene oxide [28,29], chemical vapor deposition (CVD) [30-32], carbon segregation [33,34], chemical synthesis [35,36] and deterministic placement [37] have been developed to produce single-layer 
graphene (SLG) or few-layer graphene (FLG). Current efforts in graphene synthesis are mainly focused on the control of area, structural quality, and number of layers [38].

Liquid-phase exfoliation (LPE) involves making dispersions of graphite in various solvents by ultrasonication. LPE can probably be viewed as an alternative to solid-phase micromechanical cleavage that is more facile and gives larger yields. However, it is difficult to control the number of graphene layers using LPE [27,39]. In this micromechanical approach, interaction between solvents such as $N, N$-dimethylformamide (DMF) or $N$-methylpyrrolidone and graphene may lower the energy barrier of exfoliation by providing a better match of surface energies between the two components [27]. Moreover, appropriate organic molecules (both small molecules and polymers) can also be used to help exfoliate graphite into graphene by insertion between graphite layers [40,41].

Reduction of graphene oxide (GO) is the most popular wet chemical approach to produce colloidal suspensions of graphene, which is called reduced graphene oxide (rGO) [42]. GO in aqueous solution, which is generated by oxidization (resulting in oxidation of graphite, i.e., graphite oxide) and subsequent exfoliation of graphite, can be reduced to graphene by reducing agents [43], thermal treatment [44] or electrochemical methods [45], transforming electrically nonconductive GO to conductive graphene. Because of facile solution processing, large yields, and low cost, this wet chemistry approach to graphene is especially attractive in the fields of chemistry and biomaterials. However, the transport properties of the resulting $\mathrm{rGO}$ are moderate because of the presence of defects and oxygen groups in the graphene plane.

SLG and FLG can be grown on metal substrates via CVD. When a catalytic metal surface is heated to a suitable temperature, gaseous hydrocarbons decompose into carbon atoms and hydrogen gas (or oxygen gas), and the carbon atoms then form a graphene mono- or multilayer [46]. Although large-area SLG or FLG films have been generated by CVD, and graphene-like carbon materials have been produced by substrate-free CVD, radio-frequency plasma-enhanced CVD, aerosol pyrolysis and solvothermal synthesis, the uniform growth of SLG or graphene with a predefined number of layers is still a major challenge possibly because of the large amount of carbon sources adsorbed by catalytic metals [47].

Carbon segregation has also been used to prepare graphene layers from silicon carbide $(\mathrm{SiC})$ or carbon-doped metal substrates $[33,34]$. SLG or FLG can be fabricated via sublimation of $\mathrm{Si}$ from $\mathrm{SiC}$ or catalytic decomposition of hydrocarbons following high temperature annealing. Similar to CVD, this segregation method also faces the challenge of how to achieve high quality graphene layers with accurate thickness control over an entire substrate.

In this article, we briefly review our recent work on the controlled synthesis of graphene and graphene-based composites, the development of methods to characterize graphene layers, and the use of graphene in clean energy applications and rapid DNA sequencing.

\section{Synthesis and characterization of graphene- based materials}

\subsection{Large-scale synthesis of SLG}

In contrast to $\mathrm{GO}$ and $\mathrm{rGO}$ synthesized by solution-based oxidation and reduction, graphene layers synthesized by dry methods such as CVD and surface segregation have superior electron transport characteristics and features derived from these transport properties [1,9]. The unique electronic, photonic, mechanical, and thermal properties of graphene layers are dependent on the number of layers and crystalline structure of graphene layers as well as their stacking. Although coverage of SLG grown on $\mathrm{Cu}$ foil of more than $95 \%$ has been achieved [47], this graphene is not epitaxial. The controlled synthesis of graphene with a predefined number of layers over an entire substrate is still a major challenge. Variability in the thickness of graphene layers and grains over the same substrate leads to the fluctuation of electron transport properties [30], and thus prevents us from taking advantage of the distinct properties of monolayer (ML), bilayer (BL), and trilayer (TL) graphene in practical largescale integrated applications.

Among the substrates used in the synthesis of graphene, $\mathrm{Ni}(111)$ surfaces are one of the best templates because of the small lattice mismatch of this surface with that of graphene and highly oriented pyrolytic graphite (HOPG) [48]. This makes $\mathrm{Ni}(111)$ one of the most promising catalytic metals for commensurate epitaxial growth of structurally homogeneous graphene. However, the large solubility of carbon in $\mathrm{Ni}$ makes it difficult to obtain graphene layers with uniform thickness over the whole substrate. To solve this problem and realize commensurate epitaxial growth of graphene on metal templates, we recently developed a simple method to produce SLG on a Ni film substrate [38] via surface segregation [49-51]. We first deposited a thin film of $\mathrm{Ni}(\sim 100 \mathrm{~nm}$ thick) on a HOPG(0001) surface and then treated the sample under high vacuum. During heating, the polycrystalline Ni film was transformed into a Ni(111) surface, and simultaneously carbon atoms diffused into the $\mathrm{Ni}$ template from HOPG. Finally, SLG on top of the Ni template was formed via carbon segregation during cooling. We investigated the homogeneity of the synthesized SLG by various methods including Raman spectroscopy and scanning tunneling microscopy (STM) [52-54]. All of the results suggested that the graphene layer synthesized on the whole Ni film was SLG (Figure 1).

\subsection{Auger electron characteristics of graphene layers}

As mentioned above, the optical and electronic properties of graphenes rely heavily on the number of graphene layers 

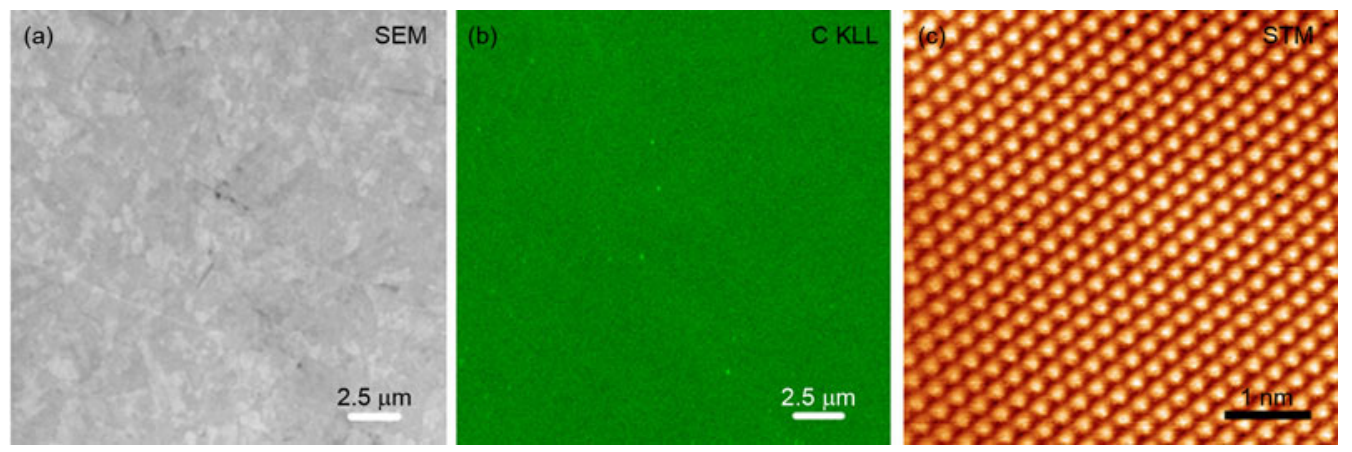

Figure 1 Extended SLG synthesized on a Ni film. (a) Scanning electron microscopy (SEM) image; (b) C KLL Auger electron map corresponding to the image in (a); and (c) typical ultrahigh vacuum STM image of graphene on a Ni film. Reprinted with permission from [38], Copyright 2011, American Chemical Society.

and their stacking, dopants, defects, and coupling with the underlying substrate. It has been pointed out that ML, BL, and TL graphenes show different electronic properties, and the electronic structure rapidly evolves with the number of layers, approaching the three-dimensional limit of graphite at $~ 10$ layers [55]. Thus, identification of the number of graphene layers, i.e., determination of the thickness of a graphene sample, is essential for informed research and distinct applications. Raman spectroscopy is a standard technique to identify graphene and count the number of graphene layers based on the features of $\mathrm{G}$ and $2 \mathrm{D}$ bands, including the intensity and shape of the peaks and the full width at half-maximum of the 2D peak [56]. However, in most cases, Raman spectroscopy is only suitable for identifying the graphene layers on $\mathrm{SiO}_{2}$ substrates with a typical thickness of $\sim 300 \mathrm{~nm}$ [57]. Also, Raman spectroscopy is not a high-resolution technique.

Recently, we established Auger electron spectroscopy (AES) as a method to characterize the number and structure of graphene layers on arbitrary substrates [58]. We found that AES gives distinct spectrum shape, intensity and energy characteristics with an increasing number of graphene layers (Figure 2). Based on a study of standard graphene sheets, we directly calculated electron inelastic mean free
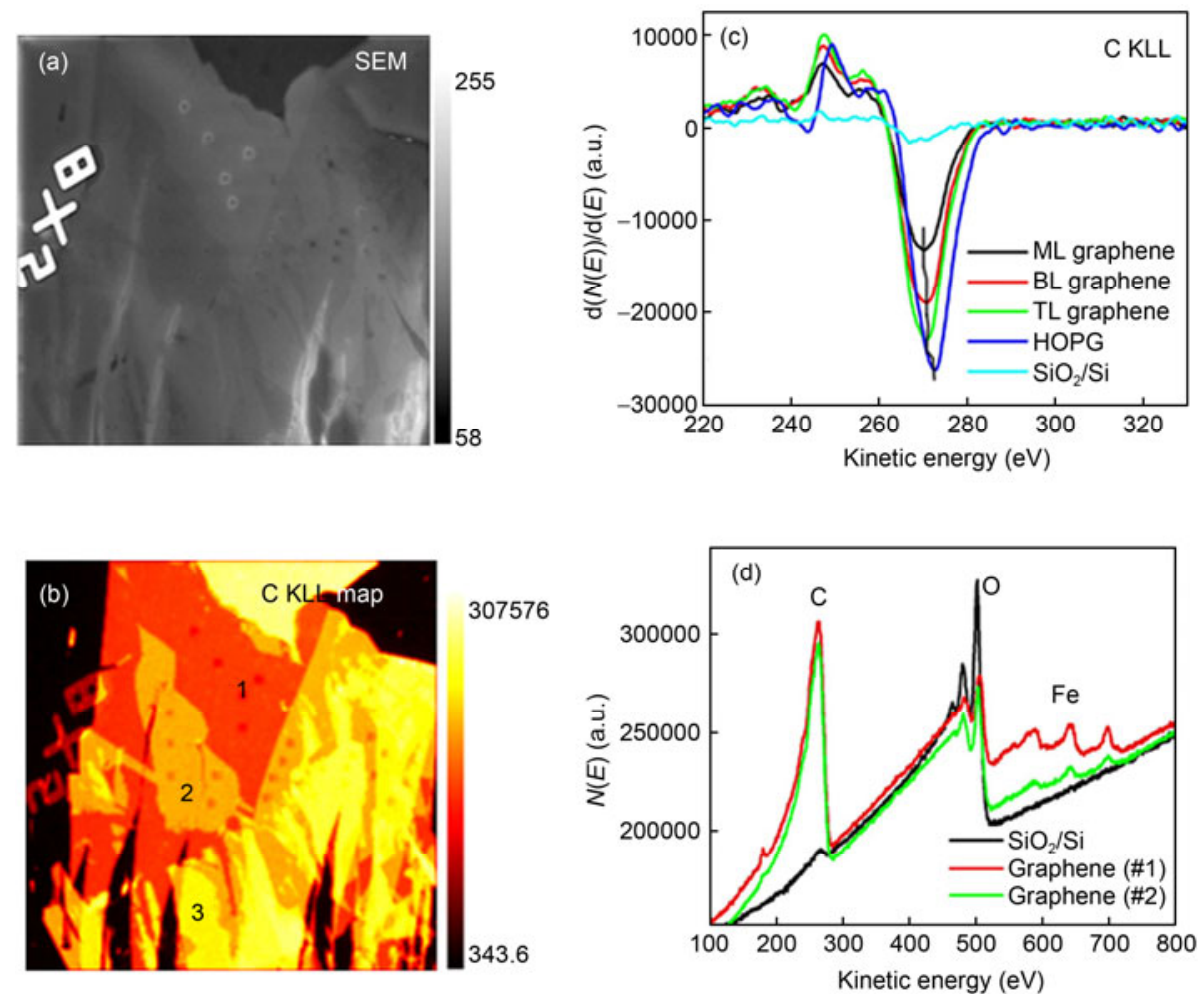

Figure 2 AES characterization of the number and structure of graphene layers. (a) $\mathrm{SEM}$ image of a graphene flake on $\mathrm{SiO}_{2}$; (b) C KLL Auger electron map corresponding to (a), showing the different color contrast of different numbers of layers; (c) AES spectra acquired for ML, BL, and TL graphene, and HOPG; (d) AES spectra of a graphene sheet transferred onto $\mathrm{SiO}_{2}$. Fe impurity from $\mathrm{FeCl}_{3}$ etchant used for remove Ni substrate was detected. Reprinted with permission from [58], Copyright 2010, American Chemical Society. 
paths (IMFP) for graphene layers. Once we know the IMFP for graphene layers, we can determine the number of graphene layers simply by measuring the peak intensity according to the electron attenuation model. AES can allow up to six layers of ML graphene on any substrate to be accurately measured. Furthermore, AES is a high-resolution technique so it can be used to study the uniformity of graphene layers at the nanoscale, allowing the resolution of defects in graphene layers [59]. Therefore, impurities and dopants in graphene layers can be detected based on Auger electron characteristics.

\subsection{Covalent surface modification of GO for graphene- based materials}

GO is a major precursor of graphene-based materials. It is easily produced by chemical oxidation and subsequent exfoliation of graphite, which is inexpensive and available in large quantities from natural and synthetic sources [28,29]. Generally, GO is synthesized by one of the three methods developed by Brodie, Staudenmaier, or Hummers [60]. All of these methods involve oxidizing graphite to various levels. In the Brodie method [61], graphite is repeatedly treated with potassium chlorate $\left(\mathrm{KClO}_{3}\right)$ and nitric acid $\left(\mathrm{HNO}_{3}\right)$. This method was modified by Staudenmaier [62], who used a mixture of sulfuric acid $\left(\mathrm{H}_{2} \mathrm{SO}_{4}\right)$ and $\mathrm{HNO}_{3}$ with $\mathrm{KClO}_{3}$. Hummers et al. [63] later developed a less hazardous and more efficient method that involves treatment of graphite with a mixture of sodium nitrate $\left(\mathrm{NaNO}_{3}\right)$, potassium permanganate $\left(\mathrm{KMnO}_{4}\right)$, and concentrated $\mathrm{H}_{2} \mathrm{SO}_{4}$. Xu et al. [64] reported that mildly oxidized $\mathrm{GO}$ can be prepared using a modified Hummers' method involving a single chemical exfoliation of graphite with a smaller amount of $\mathrm{KMnO}_{4}$. Compared with GO made by Hummers' method, the mildly oxidized GO contains fewer defects and can be used to prepare highly conductive graphene by chemical reduction. GO is a nonstoichiometric compound of carbon, oxygen and hydrogen in variable ratio, which is dependent on the synthesis method and degree of oxidation $[65,66]$. The chemical structure of GO is often assumed to be a graphene sheet bonded to oxygen-containing functionalities including epoxide and hydroxyl groups on the basal plane and carbonyl and carboxyl groups at the edges $[28,65]$. These functional groups make GO hydrophilic, which allow it to disperse effectively in some polar solvents such as water, alcohol and DMF [67]. These groups also facilitate the chemical functionalization of GO $[68,69]$.
A variety of reactions have been developed for chemical functionalization of GO. Stankovich et al. [70] successfully functionalized GO by treating it with organic isocyanates. Isocyanate treatment results in the functionalization of the carboxyl and hydroxyl groups in GO via formation of amides and carbamate esters, respectively. Such isocyanatederivatized GOs do not exfoliate in water but readily form stable dispersions in polar aprotic solvents such as DMF, which facilitates the formation of grapheme-polymer composites [42]. The coupling reaction between carboxyl groups and nucleophilic species such as amine or hydroxyl moieties can also be used to functionalize GO with activation by thionyl chloride $\left(\mathrm{SOCl}_{2}\right)$ or catalysis by carbodiimide to form amides or esters, respectively [71-73].

It has been demonstrated that epoxy groups behave as an electrophilic center in typical organic chemistry. Inspired by this, we synthesized alkyl-functionalized graphene materials by the ring-opening amination of epoxy groups on the basal plane of GO with octadecylamine (Figure 3) [74]. Octadecylamine-functionalized GO (ODA-GO) is hydrophobic and can be dispersed well in many organic solvents as single sheets. We fabricated carbon nanoscrolls (CNSs) using ODA-GO nanosheets as building blocks by compression of Langmuir-Blodgett (LB) films. In this process, a dispersion of ODA-GO in toluene was added dropwise onto a water subphase in a LB trough. After the evaporation of toluene, individual ODA-GO nanosheets floated on the water surface because of their hydrophobic nature. The scattered nanosheets were pushed together into a compact ML by two moving barriers. Further compression caused the scrolling of flexible ODA-GO nanosheets to form CNSs, as shown in Figure 4 . The scrolls can be unwound by specific solvents. Activated by thionyl chloride before amine treatment, GO can be grafted with more alkyl chains through both acylation of carboxyl groups and ring-opening amination of epoxy groups [75].

\subsection{Graphene-based hybrid nanomaterials}

Graphene has been produced efficiently in bulk amounts at low cost using chemical conversion from graphite such as by chemical reduction from GO [30]. Unlike other approaches, chemical synthesis of graphene using graphite, graphite oxide or other graphite derivatives as starting materials is not only scalable but can also produce graphene that is processable and possesses new functions. The unique two-dimensional structure as well as the extraordinary electronic,

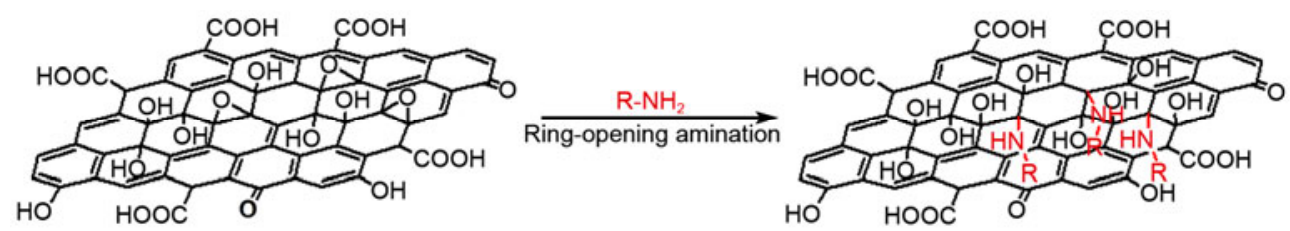

Figure 3 Ring-opening amination of epoxy groups on GO with octadecylamine. 

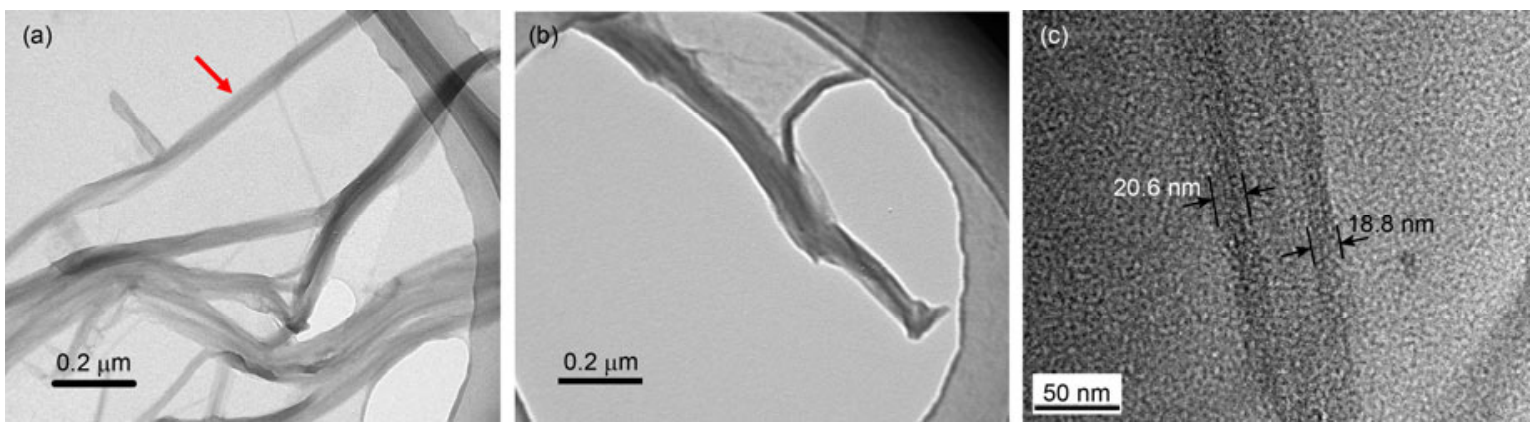

Figure 4 Typical CNS structures produced by compression of LB films. (a) TEM image of CNSs (the red arrow shows a CNS with a well-defined hollow core); (b) a half scrolled CNS without a cap at the end; (c) high-resolution TEM image of a CNS with a tubular structure. Reprinted with permission from [73], Copyright 2010, Elsevier.

optical, mechanical and chemical properties of graphene provides a robust atomic-scale scaffold for the design of new graphene-based hybrid nanomaterials [76]. For example, when decorated with metal nanoparticles, the intrinsic properties of graphene can be finely tuned to adapt varied applications such as catalysis, energy generation and storage, optoelectronics, and sensors [77]. Most hybrids reported in the literature consist of graphene with noble metal nanoparticles including $\mathrm{Au}$ [20], Pt [78,79], Pd [80] and $\mathrm{Ag}$ [81]. Fe [82], $\mathrm{Cu}$ [83], Sn [84] and Co [85] nanoparticles have also been used to fabricate composites with graphene.

Despite great progress in the synthesis of various metalgraphene composites recently, anchoring metal nanoparticles to GO or rGO surfaces to modulate electronic and other properties, improving the dispersibility of $\mathrm{rGO}$, and controlling the size, uniformity, and density of nanoparticles on the graphene planes remains a major challenge. In contrast to the unique transport properties of graphene, GO is insulating.
Randomly distributed oxygen functionalities on the surface of GO can result in inhomogeneous GO-based composites [86]. Chemical reduction of GO to rGO results in conductivity but also poor dispersibility because of $\pi$ - $\pi$ stacking interactions, which make individual $\mathrm{rGO}$ sheets almost inaccessible for modification or functionalization [74]. Currently, there are several methods used to prevent aggregation of rGO and graphene composites [87-89].

We recently developed a strategy to synthesize a waterdispersible graphene composite uniformly functionalized with gold nanodots (GNDs) [20]. The basic principle of this strategy is to use ethanethiol-perylene tetracarboxylic diimide (ETPTCDI) as an adhesive that, on one hand, uniformly assembles on graphene with its perylene $\pi$-motif and, on the other hand, bonds to the GNDs through thiol-Au interactions. ETPTCDI causes rGO to disperse well after reduction from GO-ETPTCDI, and serves as a template to orderly decorate graphene with GNDs (Figure 5). This novel in situ (a)

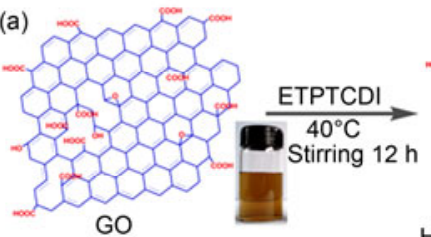

GO

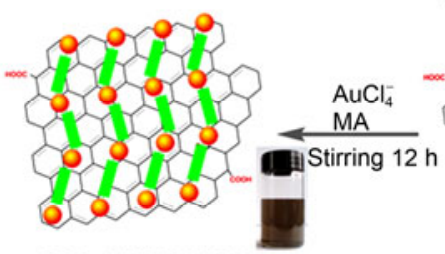

RGO-ETPTCDI-GND

$$
==\operatorname{mogh0}(\text { ETPTCDI) }
$$

$\mathrm{MA}($ Mercaptacetic acid $)=\mathrm{HS} \widetilde{O}_{\mathrm{O}}^{\mathrm{O}}$
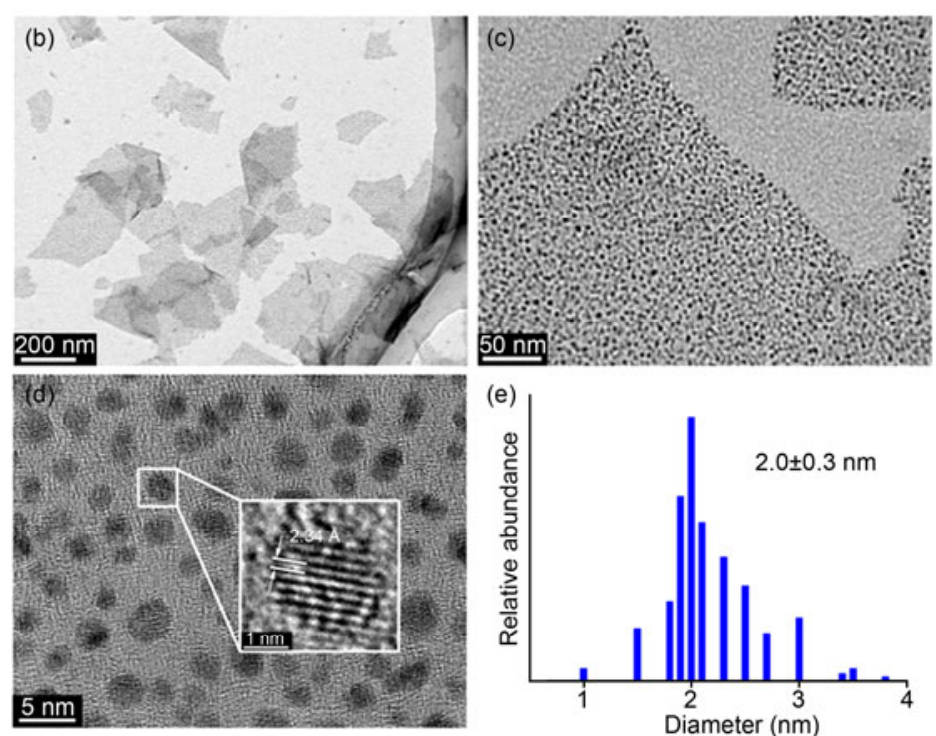

Figure 5 Uniform decoration of GNDs on rGO-ETPTCDI sheets. (a) Synthesis of rGO-ETPTCDI-GND; (b)-(d) TEM images of rGO-ETPTCDI-GND; (e) particle size distribution of Au GNDs on rGO-ETPTCDI-GND sheets estimated by measuring the size of more than 400 GNDs, expressed as mean \pm standard error. Reprinted with permission from [20], Copyright 2011, Royal Society of Chemistry. 
method enables us to produce dispersible, reliable, and processable rGO-ETPTCDI-GND sheets with improved electrocatalytic activity and varying size and density of GNDs.

We demonstrated that to attain dispersible graphenebased hybrids, aggregation of nanoparticle-crosslinked rGO must be prevented. Nanoparticles themselves generally tend to aggregate, so the nanoparticles could attach to more than one rGO sheet and thus form nanoparticle-crosslinked rGO aggregates. RGO sheets need to be well-dispersed to allow further functionalization. These two principles need to be considered to synthesize high quality graphene hybrid materials and explore further potential applications.

\section{Use of grapheme-based materials in clean energy applications and as biosensors}

\subsection{Organic photovoltaic devices}

Because of unique properties such as tunable electronic properties, optical transparency, robustness and flexibility, graphene-based materials have been widely explored for use in polymer solar cells (PSCs). It has been reported that graphene-based materials and composites can be used as transparent electrodes [90,91] and electron acceptors in PSCs [92-95].

We used GO as an interfacial layer (IFL) to modify the bulk-heterojunction (BHJ)BHJ/metal anode contact in inverted poly[3-hexylthiophene] (P3HT):[6,6]phenyl $\mathrm{C}_{61}$ butyric acid methyl ester (PCBM) PSCs [17]. GO was deposited onto the active layer simply by spin-coating a butyl alcohol solution of GO. The device containing GO exhibited a remarkable improvement in power conversion efficiency compared to the device lacking an interfacial layer, indicating that GO can effectively modify the $\mathrm{BHJ} /$ metal anode interface to facilitate hole collection. In addition, the device performance with a $\sim 2-3 \mathrm{~nm}$ thick GO layer is even better than that of a control device containing poly(3,4-ethylenedioxythiophene):poly(styrenesulfonate) (PEDOT:PSS) as the IFL. Further increase in the thickness of the GO layer will result in the decrease of the device performance with higher series resistance, which can be attributed to the high electrical resistance of GO.

To further understand the mechanism of GO as an IFL on the anode in PSCs, we investigated the chemical composition of GO by various techniques such as X-ray photoelectron spectroscopy, Fourier transform infrared spectroscopy, and $\mathrm{pH}$ titration analysis [18]. GO is acidic in nature because of the presence of chemical species such as carboxylic acid, phenolic and enolic hydroxyl groups, which can lead to proton dissociation. The proton density of GO used in this research was calculated to be one exchangeable proton per 8.7 carbon rings. We measured the electrical conductivity of the P3HT thin film before and after GO deposition by fabricating organic field-effect transistor (OFET) devices (Figure 6(a)). It was found that the electrical conductivity of the P3HT thin film increased by six orders of magnitude and exhibited metallic-like characteristics when GO was deposited on its surface. The dramatic increase in conductivity of the P3HT thin film was attributed to protonic doping of the surface of P3HT by the GO layer, which was confirmed by the observation of new charge transfer absorption peaks in the NIR region of the UV-Vis-NIR spectrum. Compared to small molecule dopants (e.g., $\mathrm{FeCl}_{3}$, $\mathrm{I}_{2}, \mathrm{HCl}$ and $\mathrm{NOPF}_{4}$ ) [96-98], the unique 2D structure of GO prevents it from penetrating into the bulk of conjugated polymers, making it an ideal material for surface doping. This finding provides a new strategy to improve the electrical contact of metal/conjugated polymer interfaces in organic electronic devices such as PSCs, organic light-emitting diodes, and OFETs. The surface doping effect was demonstrated in inverted P3HT:PCBM PSCs using metals ( $\mathrm{Au}, \mathrm{Ag}, \mathrm{Al}$ ) with different work functions (WFs) as the top anode, as shown in Figure 6(b). When a thin layer of GO is incorporated between the anode and active layer, the devices show improved performance with a open circuit voltage of $\sim 0.62 \mathrm{~V}$, independent of the WFs of the different metals. This can be attributed to the formation of an Ohmic contact between the active layer and top metal electrode caused by the surface doping of P3HT by GO [99].

\subsection{Graphene for rapid DNA sequencing}

Biosensing has the potential to improve the quality of human life. Because of their large surface area and excellent transport properties, biological applications of graphene, GO and rGO have attracted significant interest based on research experience in carbon nanotubes and various nanowires. Graphene-based materials have been used in electrochemical, impedance, and fluorescence biosensors, as well as biomolecular labels and transducers in OFETs.

The most ambitious biological application of graphene is for rapid, inexpensive electronic DNA sequencing. Affordable and rapid genome sequencing is widely regarded as the
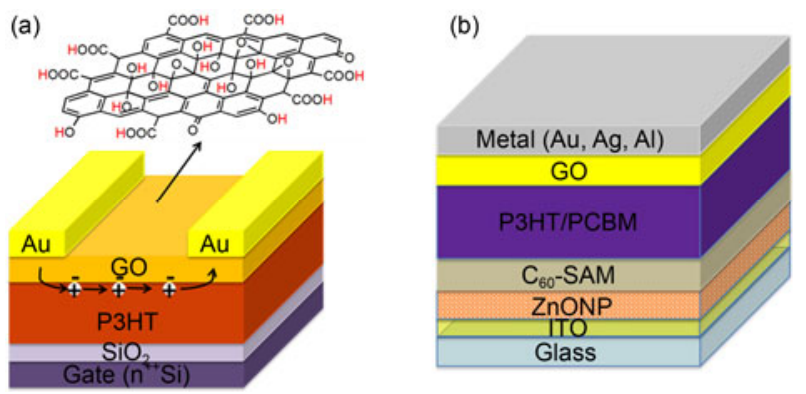

Figure 6 (a) Schematic diagrams of the cross-section of an OFET containing P3HT and GO (P3HT/GO); (b) device configuration of inverted PSCs containing GO as a hole-selective IFL and different metals as the top electrode. Reprinted with permission from [18], Copyright 2011, WILEYVCH Verlag GmbH \& Co. KGaA, Weinheim. 
next great frontier for science and will eventually revolutionize personalized and predictive medicine and custom medical treatment, enabling doctors to determine genetic susceptibility to a host of diseases and tailor therapies to an individual's genome. We proposed in 2009 that using graphene can solve the bottle-neck issue of how to achieve single-base resolution of electronic DNA sequencing (Figure 7) [100]. Our proposal was based on the following facts: the base-specific electronic signatures of the four DNA bases [101,102], and the $0.335 \mathrm{~nm}$ thickness of conducting graphene is equivalent to or less than the separation between neighboring DNA bases in a stretched single-stranded DNA chain. We further proposed to integrate the graphene sensing element with nanofluidics because it could be easy to control the movement of DNA chains and the interaction between graphene and DNA bases in a nanochannel [103]. Later numerical simulation revealed that graphene-based nanopores or nanochannels may rapidly read out sequences of individual DNA molecules with 100\% accuracy [104-106]. It has been shown experimentally that graphene nanopores can be used as subnanometer electrodes [107-109]. All of the studies to date show that graphene is promising for direct, rapid and inexpensive DNA sequencing. This is a new and rapidly expanding field, and the development of such technology would surely lead to the "\$1000 genome" and allow personalized medicine to be realized.

\section{Perspective}

Herein, we have highlighted our recent efforts to fabricate graphene-based materials and explore their application in organic photovoltaic devices and rapid electronic DNA sequencing. Because of their unique two-dimensional structure and excellent electronic, optical and mechanic properties, graphene-based materials show tremendous potential in various applications. In energy conversion, one promising

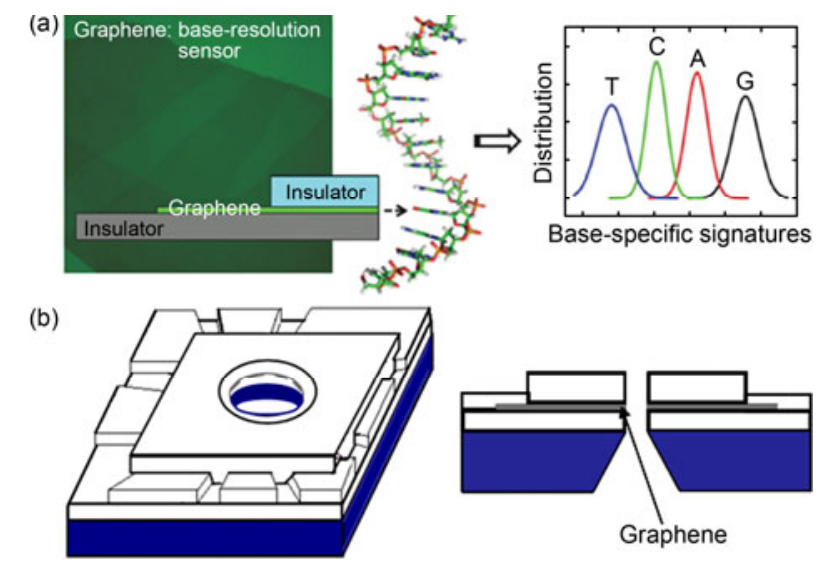

Figure 7 Graphene for DNA sequencing. (a) Basic principle of using graphene for electronic DNA sequencing with single-base resolution. Reprinted with permission from [100], Copyright 2009, WILEY-VCH Verlag $\mathrm{GmbH} \& \mathrm{Co}$. KGaA, Weinheim; (b) a graphene-based nanopore. application is exploiting graphene as a transparent conducting electrode for solar cells and displays. Compared to traditional transparent indium tin oxide films, advantages in cost, resources, and mechanical flexibility make graphene especially useful in the emerging field of flexible organic electronic devices. However, the sheet resistance of graphene electrodes synthesized by CVD remains higher than that of ITO, which is mainly caused by structural defects in the graphene films. Doping graphene can increase carrier concentration; however, the stability of dopants needs to be improved. The development of low-cost and solutionprocessable graphene-based IFLs for PSCs also has attracted great attention. Incorporation of semiconductor or metal nanoparticles into graphene-based materials can provide greater versatility in energy harvesting and conversion, and selective catalytic and sensing applications. The use of graphene-based materials to form lightweight, strong, and electrically tunable composites will also emerge in many applications. One challenge facing graphene used as an active material in PSCs is the absence of a band gap in pristine graphene.

Since researchers completed sequencing the first human genome in 2003 at a cost of about $\$ 3$ billion, the cost of DNA sequencing has been dropping steadily by using second generation sequencing platforms. Sequencing the human genome could improve our understanding of the biological functions encoded in it and the biological basis of inherited diseases and cancer. The ultimate goal is for sequencing to become so simple and inexpensive that it can be routinely employed as a general-purpose tool throughout biomedicine. To fulfill this potential, the cost of wholegenome sequencing will need to decrease to a few hundred US dollars. With new approaches under development, these goals may be feasible within the next decade. Graphenebased nanopore technology is one possible method for singlemolecule DNA sequencing at low cost and with high throughput. Despite the theoretical possibility of accurately sequencing DNA by graphene nanopores and the demonstration of DNA translocation through graphene nanopores, there are various fundamental questions about sequencing with graphene nanopores that remain. For example, the detection mechanism of the local electrical field [104,105], whether high ionic strength prevent detection of charged DNA molecules [106], how to make graphene pores with a size of $\sim 2 \mathrm{~nm}$ [104], and whether single-nucleotide resolution is possible in the presence of thermodynamic fluctuation all require further study. Without doubt, this exciting yet preliminary work will certainly be the precursor for many future studies on the use of graphene for DNA sequencing.

To move forward, controlled fabrication of graphenebased materials and the fine tuning of their properties should be further explored and exploited to meet the needs of emerging applications. Chemical exfoliation of graphite, yielding GO, provides a practical route towards low-cost 
bulk production of solution-processable graphene-based materials. Despite the random distribution of functional groups on the GO surface, the presence of such groups provides an excellent platform to tune the electronic and optical properties of graphene-based materials. In particular, the intrinsic fluorescence of GO in the visible and NIR range opens up exciting and previously unforeseen optical applications for graphene-based materials. CVD and surface segregation are two of the most promising methods to produce graphene over large areas. Besides difficulty in controlling the number and structural quality of graphene layers on a whole substrate, a challenge for graphene produced by CVD and surface segregation is reliable and scalable transfer of the fabricated graphene from the synthesized substrate to a target platform for practical applications in ultrafast optoelectronic devices, energy generation and storage, and chemical and biological sensors [110].

This work was supported by the National Natural Science Foundation of China (50990063, 50973095, and 51011130028), the Developing Program of Zhejiang Province Key Scientific and Technical Innovation Team (2009R50004), Zhejiang Provincial National Science Foundation of China (Youth Talent Program: R4110030), Science and Technology Department of Zhejiang Province (Qianjiang Talent Program: 2011R10077), and the Fundamental Research Funds for the Central Universities (2011QNA4030).

1 Geim A K, Novoselov K S. The rise of graphene. Nat Mater, 2007, 6: 183-191

2 Dreyer D R, Ruoff R S, Bielawski C W. From conception to realization: An historial account of graphene and some perspectives for its future. Angew Chem Int Ed, 2010, 49: 9336-9345

3 May J W. Platinum surface LEED rings. Surf Sci, 1969, 17: 267-270

4 Boehm H P, Setton R, Stumpp E. Nomenclature and terminology of graphite intercalation compounds. Carbon, 1986, 24: 241-245

5 Novoselov K S, Geim A K, Morozov S V, et al. Electric field effect in atomically thin carbon films. Science, 2004, 306: 666-669

6 Novoselov K S, Jiang D, Schedin F, et al. Two-dimensional atomic crystals. Proc Natl Acad Sci USA, 2005, 102: 10451-10453

7 Novoselov K S, Geim A K, Morozov S V, et al. Two-dimensional gas of massless Dirac fermions in graphene. Nature, 2005, 438: 197200

8 Zhang Y B, Tan Y W, Stormer H L, et al. Experimental observation of the quantum Hall effect and Berry's phase in graphene. Nature, 2005, 438: 201-204

9 Park S, Ruoff R S. Chemical methods for the production of graphenes. Nat Nanotechnol, 2009, 4: 217-224

$10 \mathrm{Xu} \mathrm{Y} \mathrm{X,} \mathrm{Shi} \mathrm{G} \mathrm{Q.} \mathrm{Assembly} \mathrm{of} \mathrm{chemically} \mathrm{modified} \mathrm{graphene:}$ Methods and applications. J Mater Chem, 2011, 21: 3311-3323

11 Stoller M D, Park S, Zhu Y, et al. Graphene-based ultracapacitors. Nano Lett, 2008, 8: 3498-3502

12 Bolotina K I, Sikesb K J, Jiang Z, et al. Ultrahigh electron mobility in suspended graphene. Solid State Commun, 2008, 146: 351-355

13 Lee C, Wei X, Kysar J W. Measurement of the elastic properties and intrinsic strength of monolayer graphene. Science, 2008, 321: 385388

14 Balandin A A, Ghosh S, Bao W Z. Superior thermal conductivity of single-layer graphene. Nano Lett, 2008, 8: 902-907

15 Cai W, Zhu Y, Li X, et al. Large area few-layer graphene/graphite films as transparent thin conducting electrodes. Appl Phys Lett, 2009, 95: 123115

16 Schwierz F. Graphene transistors. Nat Nanotechnol, 2010, 5: 487496
17 Gao Y, Yip H L, Hau S K, et al. Anode modification of inverted polymer solar cells using graphene oxide. Appl Phys Lett, 2010, 97: 203306

18 Gao Y, Yip H L, Chen K S, et al. Surface doping of conjugated polymers by graphene oxide and its application for organic electronic devices. Adv Mater, 2011, 23: 1903-1908

19 Ohno Y, Maehashi K, Matsumoto K. Label-free biosensors based on aptamer-modified graphene field-effect transistors. J Am Chem Soc, 2010, 132: 18012-18013

20 Yang X, Xu M S, Qiu W M, et al. Graphene uniformly decorated with gold nanodots: In situ synthesis, enhanced dispersibility and applications. J Mater Chem, 2011, 21: 8096-8103

21 Ma Y W, Zhang L R, Li J J, et al. Carbon-nitrogen/graphene composite as metal-free electrocatalyst for the oxygen reduction reaction. Chin Sci Bull, 2011, 56: 3583-3589

22 Yu D S, Dai L M. Self-assembled graphene/carbon nanotube hybrid films for supercapacitors. J Phys Chem Lett, 2010, 1: 467-470

23 Wang H W, Wu H Y, Chang Y Q, et al. Tert-butylhydroquinonedecorated graphene nanosheets and their enhanced capacitive behaviors. Chin Sci Bull, 2011, 56: 2092-2097

24 Wang Z, Tang X Z, Yu Z Z, et al. Dispersion of graphene oxide and its flame retardancy effect on epoxy nanocomposites. Chin J Polym Sci, 2011, 29: 368-376

25 Wei D C, Liu Y Q. Controllable synthesis of graphene and its applications. Adv Mater, 2010, 22: 3225-3241

26 Li X, Wang X, Zhang L, et al. Chemically derived, ultrasmooth graphene nanoribbon semiconductors. Science, 2008, 319: 1229-1232

27 Hernandez Y, Nicolosi V, Lotya M, et al. High-yield production of graphene by liquid-phase exfoliation of graphite. Nat Nanotechnol, 2008, 3: 563-568

28 Cai W W, Piner R D, Stadermann F J, et al. Synthesis and solid-state NMR structural characterization of 13C-labeled graphite oxide. Science, 2008, 321: 1815-1817

29 Eda G, Fanchini G, Chhowalla M. Large-area ultrathin films of reduced graphene oxide as a transparent and flexible electronic material. Nat Nanotechnol, 2008, 3: 270-274

30 Kim K S, Zhao Y, Jang H, et al. Large-scale pattern growth of graphene films for stretchable transparent electrodes. Nature, 2009, 457: 706-710

31 Reina A, Jia X T, Ho J, et al. Few-layer graphene films on arbitrary substrates by chemical vapor deposition. Nano Lett, 2009, 9: 30-35

32 Bae S, Kim H, Lee Y B, et al. Roll-to-roll production of 30-inch graphene films for transparent electrodes. Nat Nanotechnol, 2010, 4: 574-578

33 Sutter P W, Flege J I, Sutter E A. Epitaxial graphene on ruthenium. Nat Mater, 2008, 7: 406-411

34 Emtsev K V, Bostwick A, Horn K, et al. Towards wafer-size graphene layers by atmospheric pressure graphitization of silicon carbide. Nat Mater, 2009, 8: 203-207

35 Choucair M, Thordarson P, Stride J A. Gram-scale production of graphene based on solvothermal synthesis and sonication. Nat Nanotechnol, 2009, 4: 30-33

36 Wang X, Zhi L J, Tsao N, et al. Transparent carbon films as electrodes in organic solar cells. Angew Chem Int Ed, 2008, 47: 29902992

37 Vijayaraghavan A, Sciascia C, Dehm S, et al. Dielectrophoretic assembly of high-density arrays of individual graphene devices for rapid screening. ACS Nano, 2009, 3: 1729-1734

38 Xu M S, Fujita D, Sagisaka K, et al. Production of extended single-layer graphene. ACS Nano, 2011, 5: 1522-1528

39 Coleman J N. Liquid-phase exfoliation of nanotubes and graphene. Adv Funct Mater, 2009, 19: 3680-3695

40 An X H, Simmons T, Shah R, et al. Stable aqueous dispersions of noncovalently functionalized graphene from graphite and their multifunctional high-performance applications. Nano Lett, 2010, 10: 4295-4301

41 Liang Y T, Hersam M C. Highly concentrated graphene solutions via polymer enhanced solvent exfoliation and iterative solvent exchange. J Am Chem Soc, 2010, 132: 17661-17663 
42 Stankovich S, Dikin D A, Dommett G H B, et al. Graphene-based composite materials. Nature, 2006, 442: 282-286

43 Stankovich S, Dikin D A, Piner R D, et al. Synthesis of graphene-based nanosheets via chemical reduction of exfoliated graphite oxide. Carbon, 2007, 45: 1558-1565

44 Schniepp H C, Li J L, McAllister M J, et al. Functionalized single graphene sheets derived from splitting graphite oxide. J Phys Chem B, 2006, 110: 8535-8539

45 Zhou M, Wang Y L, Zhai Y M, et al. Controlled synthesis of largearea and patterned electrochemically reduced graphene oxide films. Chem Eur J, 2009, 15: 6116-6120

46 Coraux J, Ndiaye A T, Busse C, et al. Structural coherency of graphene on $\operatorname{Ir}(111)$. Nano Lett, 2008, 8: 565-570

47 Li X S, Cai W W, An J H, et al. Large-area synthesis of high-quality and uniform graphene films on copper foils. Science, 2009, 324: 1312-1314

48 Fujita D, Yoshihara K. Surface precipitation process of epitaxially grown graphite (0001) layers on carbon-doped nickel(111) surface. J Vac Sci Technol A, 1994, 12: 2134-2139

49 Gao J H, Fujita D, Xu M S, et al. Unique synthesis of few-layer graphene films on carbon doped Pt83Rh17 surface. ACS Nano, 2010, 4: 1026-1032

$50 \mathrm{Xu} \mathrm{M} \mathrm{S}$, Fujita D, Chen $\mathrm{H} \mathrm{Z}$, et al. Formation of monolayer and few-layer hexagonal boron nitride nanosheets via surface segregation. Nanoscale, 2011, 3: 2854-2858

51 Fujita D. Nanoscale synthesis and characterization of graphene-based objects. Sci Technol Adv Mater, 2011, 12: 044611

52 Xu M S, Endres R G, Tsukamoto S, et al. Conformation and local environment dependent conductance of DNA molecules. Small, 2005, 1: $1168-1172$

53 Xu M S, Tsukamoto S, Satomi S, et al. Conductance of single thiolated poly(GC)-poly(GC) DNA molecules. Appl Phys Lett, 2005, 87: 083902

54 Merino P, Svec M, Pinardi A L, et al. Strain-driven moire superstructures of epitaxial graphene on transition metal surfaces. ACS Nano, 2011, 5: 5627-5634

55 Partoens B, Peeters F M. From graphene to graphite: Electronic structure around the K point. Phys Rev B, 2006, 74: 075404

56 Ferrari A C, Meyer J C, Scardaci V, et al. Raman spectrum of graphene and graphene layers. Phys Rev Lett, 2006, 97: 187401

57 Calizo I, Bao W, Miao F, et al. The effect of substrates on the Raman spectrum of graphene: Graphene-on-sapphire and graphene-on-glass. Appl Phys Lett, 2007, 91: 201904

58 Xu M S, Fujita D, Gao J H, et al. Auger electron spectroscopy: A rational method for determining thickness of graphene films. ACS Nano, 2010, 4: 2937-2945

59 Xu M S, Fujita D, Hanagata N. Monitoring electron-beam irradiation effect on graphenes by temporal Auger electron spectroscopy. Nanotechnology, 2010, 21: 265705

60 Eda G, Chhowalla M. Chemically derived graphene oxide: Towards large-area thin-film electronics and optoelectronics. Adv Mater, 2010, 22: 2392-2415

61 Brodie B C. On the atomic weight of graphite. Philos Trans R Soc London, 1959, 149: 249-259

62 Staudenmaier L. Verfahren zur darstellung der graphitsaure. Ber Dtsch Chem Ges, 1898, 31: 1481-1487

63 Hummers W S, Offeman J R E. Preparation of graphitic oxide. J Am Chem Soc, 1958, 80: 1339-1339

64 Xu Y X, Sheng X, Li C, et al. Highly conductive chemically converted graphene prepared from mildly oxidized graphene oxide. J Mater Chem, 2011, 21: 7376-7380

65 Szabo T, Berkesi O, Forgo P, et al. Evolution of surface functional groups in a series of progressively oxidized graphite oxides. Chem Mater, 2006, 18: 2740-2749

66 Zhao J P, Pei S F, Ren W C, et al. Efficient preparation of large-area graphene oxide sheets for transparent conductive films. ACS Nano, 2010, 4: 5245-5252

67 Paredes J I, Villar-Rodil S, Martinez-Alonso A, et al. Graphene oxide dispersions in organic solvents. Langmuir, 2008, 24: 10560-10564
68 Dreyer D R, Park S, Bielawski C W, et al. The chemistry of graphene oxide. Chem Soc Rev, 2010, 39: 228-240

69 Loh K P, Bao Q L, Ang P K, et al. The chemistry of graphene. J Mater Chem, 2010, 20: 2277-2289

70 Stankovich S, Piner R D, Nguyen S T, et al. Synthesis and exfoliation of isocyanate-treated graphene oxide nanoplatelets. Carbon, 2006, 44: 3342-3347

71 Niyogi S, Bekyarova E, Itkis M E, et al. Solution properties of graphite and graphene. J Am Chem Soc, 2006, 128: 7720-7721

72 Veca L M, Lu F S, Meziani M J, et al. Polymer functionalization and solubilization of carbon nanosheets. Chem Commun, 2009, 25652567

73 Liu Z, Robinson J T, Sun X M, et al. PEGylated nanographene oxide for delivery of water-insoluble cancer drugs. J Am Chem Soc, 2008, 130: $10876-10877$

74 Gao Y, Chen X Q, Xu H, et al. Highly-efficient fabrication of nanoscrolls from functionalized graphene oxide by Langmuir-Blodgett method. Carbon, 2010, 48: 4475-4482

75 Mei Q S, Zhang K, Guan G J, et al. Highly efficient photoluminescent graphene oxide with tunable surface properties. Chem Commun, 2010, 7319-7321

76 Zhu Y, Murali S, Cai W W, et al. Graphene and graphene oxide: Synthesis, properties, and applications. Adv Mater, 2010, 22: 39063924

77 Kamat P V. Graphene-based nanoarchitectures: Anchoring semiconductor and metal nanoparticles on a two-dimensional carbon support. J Phys Chem Lett, 2010, 1: 520-527

78 Liu J, Fu S, Yuan B, et al. Toward a universal "adhesive nanosheet" for the assembly of multiple nanoparticles based on a protein-induced reduction/decoration of graphene oxide. J Am Chem Soc, 2010, 132: 7279-7281

79 Zhang H, Fu Q, Cui Y, et al. Fabrication of metal nanoclusters on graphene grown on Ru(0001). Chin Sci Bull, 2009, 54: 2446-2450

80 Guo S, Dong S, Wang E. Three-dimensional Pt-on-Pd bimetallic nanodendrites supported on graphene nanosheet: facile synthesis and used as an advanced nanoelectrocatalyst for methanol oxidation. ACS Nano, 2009, 4: 547-555

81 Shen J, Shi Z, Li N, et al. Facile synthesis and application of Ag-chemically converted graphene nanocomposite. Nano Res, 2010, 3: 339-349

82 Zou Y H, Liu H B, Yang L, et al. The influence of temperature on magnetic and microwave absorption properties of $\mathrm{Fe} /$ graphite oxide nanocomposites. J Magn Magn Mater, 2006, 302: 343-347

83 Hassan H M A, Abdelsayed V, Khder A E R S, et al. Microwave synthesis of graphene sheets supporting metal nanocrystals in aqueous and organic media. J Mater Chem, 2009, 19: 3832-3837

84 Wang G, Wang B, Wang X, et al. Sn/graphene nanocomposite with 3D architecture for enhanced reversible lithium storage in lithium ion batteries. J Mater Chem, 2009, 19: 8378-8384

85 Yang S, Cui G, Pang S, et al. Fabrication of cobalt and cobalt oxide/ graphene composites: Towards high-performance anode materials for lithium ion batteries. ChemSusChem, 2010, 3: 236-239

86 Goncalves G, Marques P A A P, Granadeiro C M, et al. Surface modification of graphene nanosheets with gold Nanoparticles: The role of oxygen moieties at graphene surface on gold nucleation and growth. Chem Mater, 2009, 21: 4796-4802

87 Jasuja K, Berry V. Implantation and growth of dendritic gold nanostructures on graphene derivatives: Electrical property tailoring and Raman enhancement. ACS Nano, 2009, 3: 2358-2366

88 Quintana M, Spyrou K, Grzelczak M, et al. Functionalization of graphene via 1,3-dipolar cycloaddition. ACS Nano, 2010, 4: 3527-3533

89 Liu J B, Li Y L, Li Y M, et al. Noncovalent DNA decorations of graphene oxide and reduced graphene oxide toward water-soluble metal-carbon hybrid nanostructures via self-assembly. J Mater Chem, 2010, 20: 900-906

90 Becerril H A, Mao J, Liu Z, et al. Evaluation of solution-processed reduced graphene oxide films as transparent conductors. ACS Nano, 2008, 2: 463-470

91 De S, Coleman J N. Are there fundamental limitations on the sheet 
resistance and transmittance of thin graphene films? ACS Nano, 2010, 4: 2713-2720

92 Liu Q, Liu Z F, Zhong X Y, et al. Polymer photovoltaic cells based on solution-processable graphene and P3HT. Adv Funct Mater, 2009, 19: 894-904

93 Gupta V, Chaudhary N, Srivastava R, et al. Luminscent graphene quantum dots for organic photovoltaic devices. J Am Chem Soc, 2011, 133: 9960-9963

94 Huang X, Yin Z Y, Wu S X, et al. Graphene-based materials: Synthesis, characterization, properties, and applications. Small, 2011, 7: 1876-1902

95 Guo C X, Guai G H, Li C M. Graphene based materials: Enhancing solar energy harvesting. Adv Energy Mater, 2011, 1: 448-452

96 Chen Y, Shih I, Xiao X. Effects of $\mathrm{FeCl}_{3}$ doping on polymer-based thin film transistors. J Appl Phys, 2004, 96: 454

97 Ukai S, Ito H, Marumoto K, et al. Electrical conduction of regioregular and regiorandom poly(3-hexylthiophene) doped with iodine. J Phys Soc Jpn, 2005, 74: 3314-3319

98 Maddalena F, Meijer E J, Asadi K, et al. Doping kinetics of organic semiconductors investigated by field-effect transistors. Appl Phys Lett, 2010, 97: 043302

99 Mihailetchi V D, Blom P W M, Hummelen J C, et al. Cathode dependence of the open-circuit voltage of polymer: Fullerene bulk heterojunction solar cells. J Appl Phys, 2003, 94: 6849

$100 \mathrm{Xu} \mathrm{M} \mathrm{S}$, Fujita D, Hanagata N. Perspective and challenges of emerging single-molecule DNA sequencing technologies. Small,
2009, 5: 2638-2649

101 Xu M S, Endres R G, Arakawa Y. The electronic properties of DNA bases. Small, 2007, 3: 1539-1543

102 Xu M S, Endres R G, Arakawa Y. Transverse Electronic Signature of DNA for Electronic Sequencing. Berlin: Springer-Verlag, 2007. 205-220

103 Xu M S, Fujita D, Hanagata N. Fabrication of graphene nanoribbons, graphene nanoribbon-based field-effect transistors, and its based DNA sequencing devices. Pending Patent, JP2011-45944

104 Postma H W C. Rapid sequencing of individual DNA molecules in graphene nanogaps. Nano Lett, 2010, 10: 420-425

105 Nelson T, Zhang B, Prezhdo O V. Detection of nucleic acids with graphene nanopores: $A b$ initio characterization of a novel sequencing device. Nano Lett, 2010, 10: 3237-3242

106 Min S K, Kim W Y, Cho Y, et al. Fast DNA sequencing with a graphene-based nanochannel device. Nat Nanotechnol, 2011, 6: $162-165$

107 Garaj S, Hubbard W, Reina A, et al. Graphene as a subnanometre trans-electrode membrane. Nature, 2010, 467: 190-193

108 Merchant C A, Healy K, Wanunu M. DNA translocation through graphene nanopores. Nano Lett, 2010, 10: 2915-2921

109 Schneider G F, Kowalczyk S W, Calado V E. DNA translocation through graphene nanopores. Nano Lett, 2010, 10: 3163-3167

110 Wang Y, Zheng Y, Xu X F, et al. Electrochemical delamination of CVD grown graphene film: Toward the recyclable use of copper catalyst. ACS Nano, 2011, 5: 9927-9933

Open Access This article is distributed under the terms of the Creative Commons Attribution License which permits any use, distribution, and reproduction in any medium, provided the original author(s) and source are credited. 